\title{
Repetitive Transcranial Magnetic Stimulation Over the Left Posterior Middle Temporal Gyrus Reduces Wrist Velocity During Emblematic Hand Gesture Imitation
}

\author{
Arran T. Reader ${ }^{1,2} \cdot$ Nicholas P. Holmes ${ }^{3}$ \\ Received: 28 June 2018 / Accepted: 26 October 2018 / Published online: 8 November 2018 \\ (c) The Author(s) 2018
}

\begin{abstract}
Results from neuropsychological studies, and neuroimaging and behavioural experiments with healthy individuals, suggest that the imitation of meaningful and meaningless actions may be reliant on different processing routes. The left posterior middle temporal gyrus (pMTG) is one area that might be important for the recognition and imitation of meaningful actions. We studied the role of the left pMTG in imitation using repetitive transcranial magnetic stimulation (rTMS) and two-person motion-tracking. Participants imitated meaningless and emblematic meaningful hand and finger gestures performed by a confederate actor whilst both individuals were motion-tracked. rTMS was applied during action observation (before imitation) over the left pMTG or a vertex control site. Since meaningless action imitation has been previously associated with a greater wrist velocity and longer correction period at the end of the movement, we hypothesised that stimulation over the left pMTG would increase wrist velocity and extend the correction period of meaningful actions (i.e., due to interference with action recognition). We also hypothesised that imitator accuracy (actor-imitator correspondence) would be reduced following stimulation over the left pMTG. Contrary to our hypothesis, we found that stimulation over the pMTG, but not the vertex, during action observation reduced wrist velocity when participants later imitated meaningful, but not meaningless, hand gestures. These results provide causal evidence for a role of the left pMTG in the imitation of meaningful gestures, and may also be in keeping with proposals that left posterior temporal regions play a role in the production of postural components of gesture.
\end{abstract}

Keywords Action recognition $\cdot$ Apraxia $\cdot$ Dual-route $\cdot$ Kinematics $\cdot$ Semantic storage $\cdot$ Two-person

Handling Editor: Micah M. Murray.

Electronic supplementary material The online version of this article (https://doi.org/10.1007/s10548-018-0684-1) contains supplementary material, which is available to authorized users.

Arran T. Reader

arran.reader@ki.se

1 Department of Neuroscience, Karolinska Institutet, Stockholm, Sweden

2 Centre for Integrative Neuroscience and Neurodynamics, School of Psychology and Clinical Language Sciences, University of Reading, Reading, UK

3 School of Psychology, University of Nottingham, Nottingham, UK

\section{Introduction}

The lateral posterior temporal lobe, closely bordering the occipital lobe, is associated with high level visual perception, including the recognition of biological motion, tools, and body parts (Lingnau and Downing 2015). The left temporal lobe, particularly the middle temporal gyrus, is often considered to play a role in semantic storage (Binder et al. 2009) and retrieval (Davey et al. 2015), and damage to the left posterior middle temporal gyrus (pMTG) can result in apraxia (Buxbaum et al. 2014) or deficits in action recognition (Kalénine et al. 2010; Tarhan et al. 2015).

Action recognition may be particularly important for the imitation of meaningful actions, since our ability to imitate actions we are familiar with may rely on their extraction from long-term memory (Press and Heyes 2008; Tessari and Rumiati 2004). With this in mind, the left pMTG could be particularly important for meaningful action imitation. Notably, the pMTG is frequently reported in neuroimaging 
studies of imitation (Caspers et al. 2010), and might also be involved in recognising or knowing the meaning of observed intransitive actions (Kubiak and Króliczak 2016; Möttönen et al. 2016; Villarreal et al. 2008) and distinguishing between observed social and non-social actions (Wurm et al. 2017). Conversely, the left parietal lobe may be more important for meaningless action imitation (Buxbaum and Randerath 2018; Buxbaum et al. 2014; Goldenberg 2009; Rumiati et al. 2009).

In a previous experiment (Reader et al. 2018a), in which we motion-tracked participants' wrist movements during meaningless and emblematic meaningful gesture imitation, we observed that the imitation of meaningless gestures was associated with a longer correction period than the imitation of meaningful gestures. That is, the deceleration phase of their wrist movements was longer, as reflected by a relatively earlier time to peak velocity (TPV/MT) and time to peak deceleration (TPD/MT), despite a longer overall movement time (MT). Participants also increased the speed (peak velocity, PV) of their movements during meaningless actions, perhaps reflecting a strategy designed to ensure time for a longer correction period when imitating actions that were unfamiliar (under time constraints). These results suggested that meaningful and meaningless action imitation can be distinguished by coarse-grain (wrist) kinematics. This is in keeping with neuropsychological reports (Petreska et al. 2007; Rumiati et al. 2009; Tessari and Cubelli 2014), neuroimaging (Decety et al. 1997; Menz et al. 2009; Peigneux et al. 2004; Rumiati et al. 2005; Tanaka et al. 2001), and other behavioural studies (Carmo and Rumiati 2009; Press and Heyes 2008; Tessari and Rumiati 2004) of imitation, which suggest that meaningful and meaningless action imitation may be reliant on separate routes. The modulation of these correction time parameters is likely to be strongly reliant on accurate apprehension of the observed action prior to imitation-whether it is meaningful or meaningless.

We hypothesised that repetitive transcranial magnetic stimulation (rTMS) over the left pMTG during action observation would influence kinematic correction time markers during subsequent imitation. Specifically, we hypothesised that, following stimulation over the pMTG, participants would perform meaningful actions more like meaningless actions (i.e., with earlier TPV/MT, TPD/MT, and greater $\mathrm{PV}$ and MT). We also expected that by stimulating over this area, we would reduce participant performance more in meaningful, than in meaningless, action imitation. This would be reflected in reduced imitation performance accuracy-the correspondence or correlation between the actor's and the imitator's behaviour.

\section{Materials and Methods}

\section{Participants}

We recruited 12 right-handed participants from the University of Nottingham and the surrounding area (mean \pm SE age $=24.0 \pm 1.04$ years, 1 male). The experimental procedures were approved by the local ethics committee (ref: SoPEC 904); participants gave written, informed consent; and the experiments were conducted in accordance with the Declaration of Helsinki (as of 2008).

\section{Materials and Stimuli}

The position of the participant's right arm and hand and a confederate's left arm and hand were recorded continuously using a wired Polhemus Liberty (Polhemus Inc., Colchester, VT, USA) $240 \mathrm{~Hz}, 16$ channel (8 per person) motion-tracking system with 6 degrees of freedom (x, y, $\mathrm{z}$, azimuth, elevation, and roll). Trackers were attached to the shoulder (acromial end of clavicle), elbow (olecranon), wrist (pisiform), and the tips of the thumb and fingers. Tracking points were attached using adhesive medical tape or Velcro ${ }^{\mathrm{TM}}$.

TMS was applied using a Magstim Rapid ${ }^{2}$ (The MagStim Company, Cardiff, UK) with one of two $75 \mathrm{~mm}$ outer diameter figure-of-eight precision coils. For recording motor-evoked potentials to find the resting motor threshold (RMT) in the first dorsal interosseus (FDI), and as a safety measure during stimulation (i.e., to monitor for potential seizure-related activity, Rossi et al. 2009), muscle activity was recorded continuously over the right FDI and brachioradialis using an AD Instruments Powerlab 16/30 sampling at $2 \mathrm{kHz}$ via a Dual Bioamp/stimulator and LabChart software, with $10-500 \mathrm{~Hz}$ bandpass filtering.

The experiment was controlled and data were acquired using custom software written in Labview (National Instruments). We used LabMan (custom in-house software) to document experiments, and the HandLabToolbox (available from https://github.com/TheHandLab), and MATLAB 2016b (Mathworks, Inc.) to pre-process data.

A total of 24 gestures were used as stimuli. This included 4 meaningful hand gestures ("salute", "shock", "looking into the distance", "stop"), 4 meaningful finger gestures ("okay", "silence", "thumbs up", "gun"), and 16 matched meaningless gestures (Fig. 1a, b). In the case of finger gestures, the matching was done by changing the fingers used to create the gesture and/or the orientation or position of the hand. In the case of hand gestures, matching was done by either changing the orientation or position of the hand. In all analyses we used action effector (hand/finger) as a separate factor, since 
A

\section{salute}

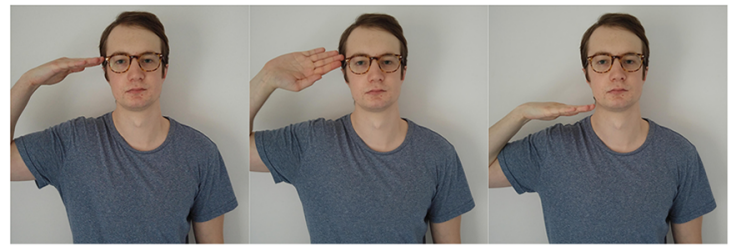

looking into the distance

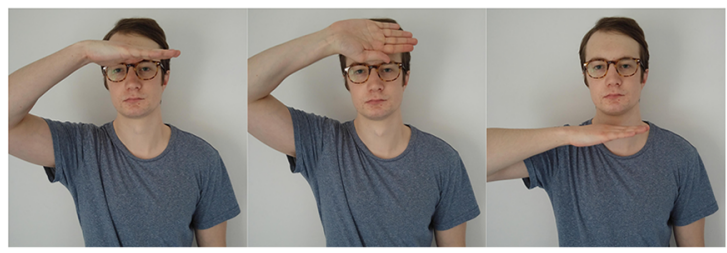

B

okay

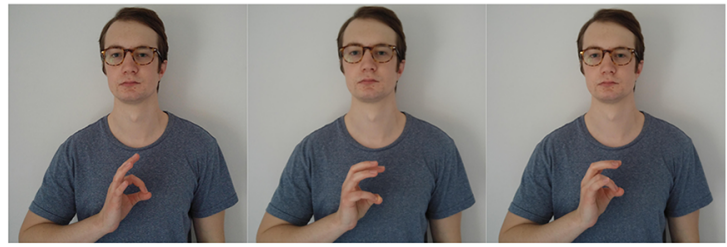

thumbs up

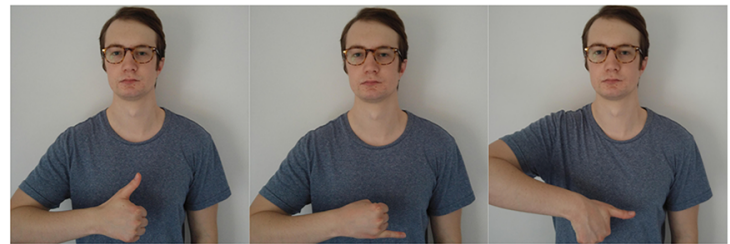

C

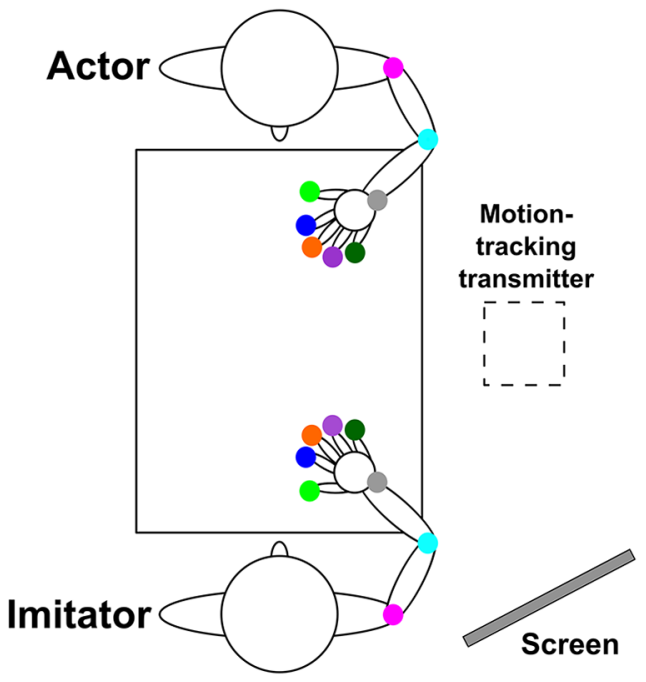

Fig. 1 Stimuli, experimental setup, and rTMS site. a Hand gesture stimuli. For each meaningful gesture, two matched meaningless gestures were created. b Finger gesture stimuli. c Experimental set-up. Dots indicate the location of motion trackers. The tracking box was shock

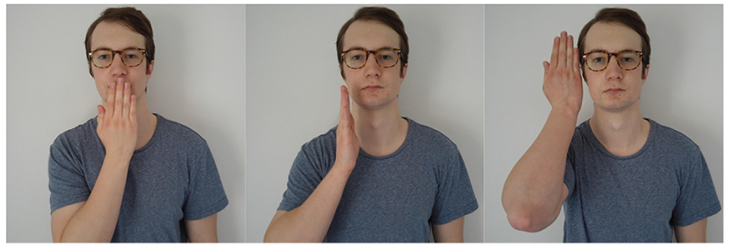

stop

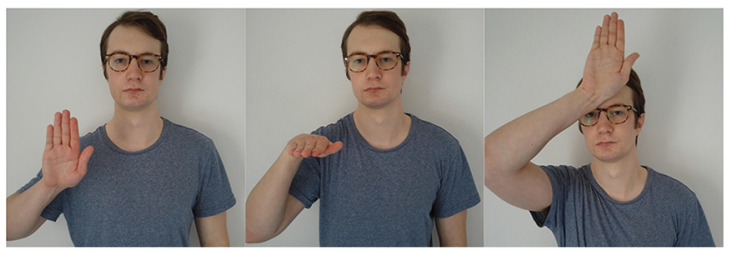

\section{silence}

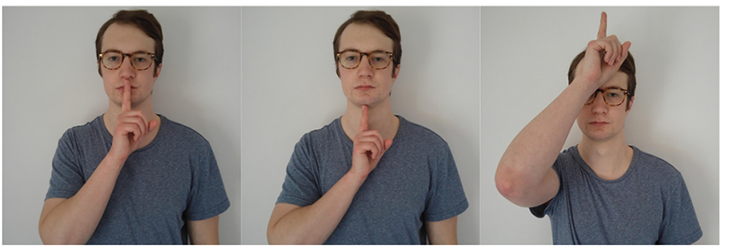

gun

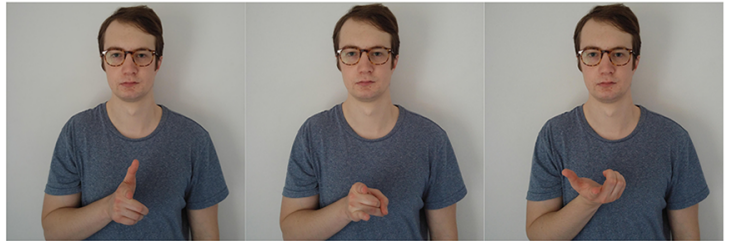

D

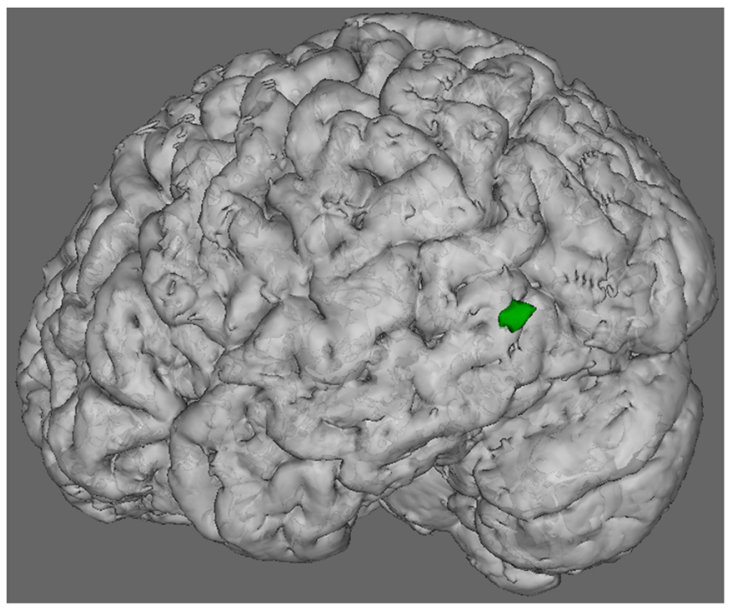

placed next to the table, and the confederate actor's actions were cued through images displayed on a computer screen that was not observable to the participant imitator. d 95\% confidence ellipsoid for the pMTG rTMS target site shown on a representative participant's brain 
previous results (Reader and Holmes 2018) suggest that our hand gesture stimuli are generally imitated more accurately than the finger gestures.

During the imitation task, participants sat opposite a confederate actor at a rectangular plastic table, approximately $76 \mathrm{~cm}$ away from each other (Fig. 1c). A start point was located $20 \mathrm{~cm}$ away from each individual using Blu Tack®. In order to inform the confederate actor of the action they needed to perform, a computer screen was placed behind the imitator. This was unobservable by the participant imitator.

\section{Selection of TMS Sites}

Visualisation of the participant's brain was performed using T1-weighted MR images alongside the BrainSight stereotactic system (Rogue Research Inc., Montreal, QC, Canada). pMTG location was based on individual neuroanatomy rather than a statistical approach (Fig. 1d). The posterior limit of the MTG was designated by drawing an imaginary line from the pre-occipital notch vertically in the dorsal direction. The stimulation site was located halfway between the superior and middle temporal sulci, and approximately $10 \mathrm{~mm}$ from the posterior limit of the MTG. A control vertex stimulation site was found using normal measures (i.e., halfway between both the two pre-auricular points and the inion and nasion).

\section{TMS Parameters}

Biphasic repetitive transcranial magnetic stimulation (rTMS) was applied over the pMTG and the vertex control for $2.67 \mathrm{~s}$ per trial at $3 \mathrm{~Hz}$ (i.e., nine pulses) and $110 \%$ of distance adjusted RMT (Stokes et al. 2007). For both stimulation sites, the coil was placed tangential to the skull. During pMTG stimulation, the coil was oriented between $\sim 22.5^{\circ}$ and $\sim 67.5^{\circ}$ in the sagittal plane (handle towards the back of the participant's head), in order to ensure tangential placement dependent on the shape of each individual participant's skull. During vertex stimulation, the handle was pointed towards the back of the participant's head. Coil position was maintained throughout the experiment by securing it to a multi-joint arm attached to the ceiling.

Experimental stimulation intensity was limited to $80 \%$ of maximum stimulator output (MSO) to reduce coil overheating. RMT was obtained using the Rossini et al. (1994) method at the start of the first session, whilst participants were seated and relaxed. Mean \pm SE RMT was $66 \pm 2.9 \%$ MSO. To create the distance adjusted RMT, the distance from M1 and pMTG to the outside of the skull was measured using the BrainSight neuronavigation software. Vertex stimulation intensity was the same as pMTG. Mean \pm SE experimental stimulation intensity was $66 \pm 2.6 \% \mathrm{MSO}$.

\section{Design and Procedure}

Participants took part in two sessions at least $24 \mathrm{~h}$ apart. In each session a single brain region was stimulated, with the order counterbalanced across participants. In every session, participants took part in both meaningful and meaningless action imitation tasks: one block of meaningful action imitation, and one block of meaningless action imitation. The block order was counterbalanced across sessions and participants. Meaningless and meaningful actions were presented in separate blocks, since there is some evidence to suggest that performing novel and known actions in a sequence recruits a single processing route, whilst presenting them separately recruits separate routes (Tessari and Cubelli 2014; Tessari and Rumiati 2004, but see; Press and Heyes 2008; Reader et al. 2018a). Hand and finger gestures were pseudorandomly interleaved within each separate block of action meaning. A trained male and female confederate were used, with each participant being assigned to one confederate for both of their testing sessions.

Both confederate actor and participant imitator began with their thumb and forefinger gripping their start points. In both meaningful and meaningless imitation tasks, action images were presented, in a random order, on a computer screen visible to the confederate but not the participant (Fig. 1c), which informed the confederate of which action to perform. A tone $1000 \mathrm{~ms}$ after the start of the image presentation signalled the actor to begin the action, which they performed and maintained until a second, lower pitched tone was played $2000 \mathrm{~ms}$ later. The actor then returned their hand to the start point. $1000 \mathrm{~ms}$ after the signal for the actor to return their hand, a higher pitched tone played to signal the imitator to copy the action, which they performed and maintained until a second, lower pitched tone was played $2000 \mathrm{~ms}$ later. The imitator then returned their hand to the start point. 64 trials were presented in this way, and the imitator was provided with a break at the halfway point. rTMS occurred during action observation, beginning $333 \mathrm{~ms}$ after the point at which the new image appeared on the screen. There was a $10 \mathrm{~s}$ gap between the end of one, and the start of the following train of stimulation, with trial timings matched to this criterion.

Following the completion of all TMS sessions, participants were presented with a questionnaire featuring the meaningful and meaningless images in a pseudorandom order. They were asked to state whether they thought each gesture had a meaning or not, and if it did to provide a brief explanation of the gesture. The purpose of this was to exclude participants if they were less than $60 \%$ consistent with our own categorisation of the actions, but no participants were excluded based on this criterion. Mean $\pm \mathrm{SE}$ percentage agreement between participants and the experimenters' categorisation was $86.5 \pm 3.25 \%$ for meaningful 
actions and $82.8 \pm 2.89 \%$ for meaningless actions. More specifically, the percentage agreement between participants and experimenters' categorisation was $75.0 \pm 5.33 \%$ for meaningful hand gestures, $97.9 \pm 2.08 \%$ for meaningful finger gestures, $78.1 \pm 3.81 \%$ for meaningless hand gestures, and $87.5 \pm 3.44 \%$ for meaningless finger gestures.

\section{Data Analysis}

Raw data are available from the Open Science Framework (https://doi.org/10.17605/OSF.IO/EGBTR). An automated script was used for pre-processing and extraction of variables. The analysis routines processed the position data from each trial of each participant and rejected artefacts. Single timepoint spikes ( $>3$ SD from the within-trial mean), in each trial's double-differentiated time-series were deemed electromagnetic artefacts and removed by interpolation across three adjacent samples either side.

The data were filtered with a bidirectional low-pass 4 th order Butterworth filter (cutoff frequency $12 \mathrm{~Hz}$ ). Trials in which either the actor or imitator moved for less than $400 \mathrm{~ms}$, started before the starting tone, or failed to finish the action before the end of the trial, were excluded. Finally, all trials were visually inspected for remaining artefacts and excluded if any remained. Following the above exclusions, a total of $78.7 \%$ of trials were maintained for statistical analysis.

In keeping with Reader et al. (2018a), four imitator wrist kinematic variables were extracted: MT, PV, TPV/MT, and TPD/MT. The mean values of these variables across every trial for each condition were analysed using repeated-measures ANOVAs with three levels: stimulation site (pMTG, vertex), action meaning (meaningful, meaningless), and action effector (hand, finger). Bonferroni-correction was used for paired comparisons, when necessary, in the event of statistically significant interactions. Since some single trackers (other than the wrist) had remaining artefacts, we removed these trackers trial-wise in each instance. This resulted in $157(0.81 \%)$ tracker-specific time-series removed from the analysis outlined below.

To test imitation accuracy, we compared the actor and the imitator 3D velocity (i.e., the change in 3D position) for each of the trackers over their primary movement (movement onset to gesture completion). To do this we ran a crosscorrelation analysis between the original actor and imitator velocity curves for each trial and for each tracker, across lags of the difference between the actor and imitator timeseries length (i.e., if actor movement duration was 180 samples, and imitator movement duration was 240 samples, then cross-correlation was performed over lags of 1 sample steps from -60 to +60 samples). From this information we took the maximum r-value and the associated lag (i.e., the point at which the imitator's velocity profile was best correlated with that of the actor) for each trial. To allow parametric analysis the resulting $\mathrm{r}$-values were converted to $\mathrm{Z}$-values using the Fisher transformation $(Z=0.5 * \ln (1+r / 1-r)$, where $\ln$ is the natural logarithm). The means of the $\mathrm{Z}$-values and lags (in milliseconds) for each condition were analysed using two three-way (stimulation site, meaning, effector) repeated measures ANOVAs.

To reduce our likelihood of reporting false positives in the multi-tracker Z-value and lag analyses, we divided our alpha value cutoff for assessing statistical significance by 8 (the number of trackers). Therefore in the ANOVAs of maximum Z-value and associated lag for each tracker, the alpha used to determine a significant result was reduced from .05 to .00625 .

\section{Results}

\section{Wrist Kinematics}

We observed a statistically significant site*meaning*effector interaction in wrist $\mathrm{PV}\left[\mathrm{F}(1,11)=8.36, \mathrm{p}=.015, \mathrm{n}^{2}=.432\right]$ (Fig. 2). We examined this statistically significant interaction by comparing PV at the level of stimulation site and action meaning using eight two-tailed paired $t$ tests. We used a Bonferroni-corrected alpha of .00625 in order to assess statistical significance for these paired comparisons.

In support of pMTG involvement in meaningful action imitation, but not in keeping with our original hypothesis, we found that wrist PV was significantly reduced for meaningful hand gestures following stimulation over the pMTG compared to over the vertex $[\mathrm{t}(11)=-4.15, \mathrm{p}=.002$, $\left.\mathrm{g}_{\mathrm{rm}}=0.497\right]$. In addition, $\mathrm{PV}$ was significantly reduced for meaningful hand gestures compared to meaningless hand gestures following pMTG stimulation $[\mathrm{t}(11)=-4.74$, $\left.\mathrm{p}<.001, \mathrm{~g}_{\mathrm{rm}}=0.555\right]$. This pattern of effects was observed in 10 out of 12 of participants. Importantly, we did not observe a significant site*meaning*effector interaction for the actor's wrist PV $\left[\mathrm{F}(1,11)=2.54, \mathrm{p}=.139, \mathrm{\eta}^{2}=.188\right]$, suggesting that the effects of rTMS could not necessarily be explained by biased actor behaviour. Furthermore, participant-wise confederate behaviour in the same direction of the statistically significant paired imitator effects was only observed in four cases.

There was no significant difference in wrist PV between pMTG and vertex stimulation for meaningful finger gestures $\left[\mathrm{t}(11)=-0.884, \mathrm{p}=.396, \mathrm{~g}_{\mathrm{rm}}=0.161\right]$, meaningless hand gestures [t(11) $\left.=-0.756, \mathrm{p}=.466, \mathrm{~g}_{\mathrm{rm}}=0.107\right]$, or meaningless finger gestures $[\mathrm{t}(11)=-0.690, \mathrm{p}=.505$, $\left.\mathrm{g}_{\mathrm{rm}}=0.141\right]$. There was no significant difference in PV between meaningful and meaningless finger gestures following rTMS over the pMTG $[\mathrm{t}(11)=-0.610, \mathrm{p}=.554$, $\left.\mathrm{g}_{\mathrm{rm}}=0.103\right]$. There was also no significant difference in 


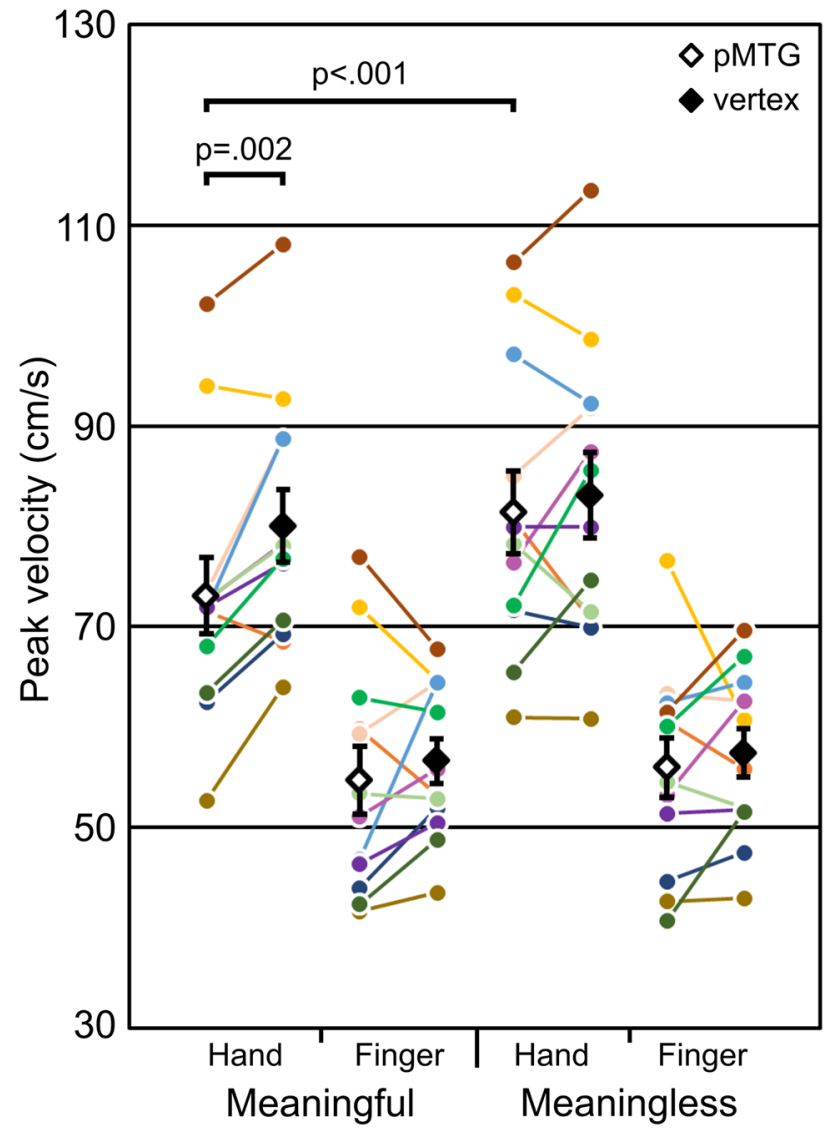

Fig. 2 Paired comparisons for imitator wrist peak velocity. Diamonds indicate mean values; error bars indicate between-participant standard error; coloured circles indicate individual participant values for each condition

PV between meaningful and meaningless finger gestures following rTMS over the vertex $[\mathrm{t}(11)=-0.785, \mathrm{p}=.449$, $\left.\mathrm{g}_{\mathrm{rm}}=0.0888\right]$, or in PV between meaningful and meaningless hand gestures following rTMS over the vertex $\left[\mathrm{t}(11)=-2.34, \mathrm{p}=.039, \mathrm{~g}_{\mathrm{rm}}=0.182\right]$.

Since the statistically significant effects of rTMS over pMTG on wrist velocity were only observed for hand gestures, we wanted to ensure that this was not just because the wrist tracker better characterised coarse-grain hand movements. We therefore decided post-hoc to also examine PV for the digits. In order to do this we subtracted the wrist position at each time point from the digit positions at each time point, in order to assess solely the digit movement element during the MT of each trial. We took the mean digit PV (i.e., across the five digits) for each condition and for each participant, and performed a repeated measures ANOVA as reported above. However, we found no statistically significant site*meaning*effector interaction, as had been observed for wrist PV (Supplemental Tables 1 and 2). We did, however, observe that the digits moved significantly slower in finger gestures [mean $\pm \mathrm{SE}$ mean digit $\mathrm{PV}=66.3 \pm 3.17 \mathrm{~cm} / \mathrm{s}]$ compared to hand gestures $(72.6 \pm 4.96 \mathrm{~cm} / \mathrm{s}), \mathrm{F}(1,11)=5.94, \mathrm{p}=.033, \mathrm{\eta}^{2}=.350$. This was probably because finger gestures required more controlled positioning of the digits.

Beyond the effects related to stimulation site, we also observed that, compared to meaningful actions, meaningless actions had a significantly smaller wrist TPV/MT and TPD/ MT, along with a significantly greater wrist PV and MT (Table 1). This replicates our previous findings (Reader et al. 2018a), which indicate that meaningless action imitation is associated with an increase in velocity in order to maintain a greater part of the movement in the correction phase, i.e., following peak deceleration.

Compared to finger gestures, hand gestures had a significantly greater wrist PV and MT, and significantly smaller TPV/MT (Table 1), suggesting, generally, that during hand gestures the wrist moved with a higher speed but for a longer duration. Statistically significant meaning*effector interactions (Supplemental Table 4) were also observed in PV $\left[\mathrm{F}(1,11)=25.3, \mathrm{p}<.001, \mathrm{\eta}^{2}=.697\right]$, TPV/MT $\left[\mathrm{F}(1,11)=6.93, \mathrm{p}=.023, \eta^{2}=.386\right]$ and $\mathrm{MT}[\mathrm{F}(1,11)=27.8$, $\left.\mathrm{p}<.001, \mathrm{\eta}^{2}=.717\right]$. We examined these statistically significant interactions using two-tailed paired $t$ tests.

Wrist PV was significantly greater in meaningless hand $(82.3 \pm 4.04 \mathrm{~cm} / \mathrm{s})$ compared to meaningful hand $(76.6 \pm 3.63 \mathrm{~cm} / \mathrm{s})$ gestures $[\mathrm{t}(11)=5.50, \mathrm{p}<.001$, $\left.\mathrm{g}_{\mathrm{rm}}=0.367\right]$. However, there was no significant difference between meaningless finger $(56.7 \pm 2.48 \mathrm{~cm} / \mathrm{s})$ and meaningful finger $(55.7 \pm 2.63 \mathrm{~cm} / \mathrm{s})$ gestures [ $\mathrm{t}(11)=0.996, \mathrm{p}=.341$, $\left.\mathrm{g}_{\mathrm{rm}}=0.105\right]$. Wrist TPV/MT was significantly smaller in meaningless hand $(.381 \pm .00754)$ compared to meaningful hand $(.412 \pm .00943)$ gestures $[\mathrm{t}(11)=-7.07, \mathrm{p}<.001$, $\left.\mathrm{g}_{\mathrm{rm}}=0.870\right]$. There was no significant difference in TPV/MT between meaningless finger $(.415 \pm .00745)$ and meaningful finger $(.426 \pm .00703)$ gestures $[\mathrm{t}(11)=-2.04, \mathrm{p}=.066$, $\left.\mathrm{g}_{\mathrm{rm}}=0.407\right]$. Finally, wrist MT was significantly greater in meaningless hand $(897 \pm 23.4 \mathrm{~ms})$ compared to meaningful hand $(833 \pm 23.0 \mathrm{~ms})$ gestures $[\mathrm{t}(11)=6.48, \mathrm{p}<.001$, $\left.\mathrm{g}_{\mathrm{rm}}=0.733\right]$. However, there was no significant difference between meaningless finger $(750 \pm 19.0 \mathrm{~ms})$ and meaningful finger $(736 \pm 19.0 \mathrm{~ms})$ gestures $[\mathrm{t}(11)=1.51, \mathrm{p}=.161$, $\left.\mathrm{g}_{\mathrm{rm}}=0.188\right]$. These results suggest that the significant main effects of action meaning in wrist PV, TPV/MT, and MT may have been driven by differences between meaningful and meaningless hand gestures.

\section{Actor-Imitator Correspondence}

There were significant main effects (Supplemental Table 5), but not interactions (Supplemental Table 6) for maximum $\mathrm{Z}$-value, representing the correlation between actor and imitator tracker velocity profiles. Hand gestures were significantly better correlated between actor and imitator than 


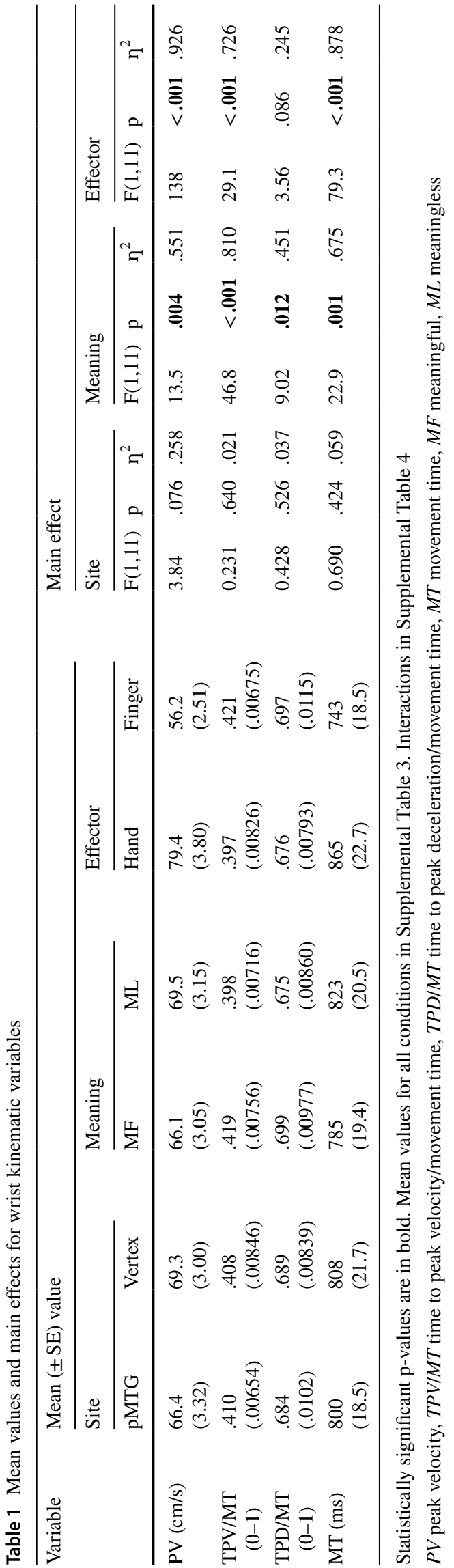

finger gestures in most trackers: shoulder, elbow, wrist, thumb, index, and little fingers (in all cases $\mathrm{F}(1,11)>18$, $\mathrm{p} \leq .001, \mathrm{n}^{2}>.600$ ). These effects, suggesting greater imitation accuracy for our hand gestures, replicate previous work (Reader and Holmes 2018). Meaningless gestures were significantly less correlated than meaningful gestures in the little finger $\left[F(1,11)=15.5, \mathrm{p}=.002, \eta^{2}=.585\right]$, but this statistically significant effect was not found in any other trackers. There were no significant main effects (Supplemental Table 7) or interactions (Supplemental Table 8) observed for lag at maximum Z-value.

\section{Discussion}

In this experiment we examined the role of the left pMTG in meaningful and meaningless action imitation. We hypothesised that rTMS over the left pMTG during action observation would impair the recognition function associated with this region, and cause participants to perform meaningful actions more akin to meaningless actions (i.e., with a longer correction period, reflected in a proportionally earlier wrist PV and peak deceleration, and greater MT and PV). In addition, we expected that, following stimulation over the pMTG, participants would show reduced accuracy in meaningful, but not meaningless, action imitation, which would be reflected in reduced actor-imitator correspondence. We did not observe results in support of either of these hypotheses. Instead, we found that stimulation over the left pMTG reduced the wrist velocity with which participants imitated meaningful hand gestures.

\section{pMTG and Meaningful Hand Gestures}

Our most notable finding was that stimulation over the left pMTG resulted in a significantly reduced wrist speed (PV) for the performance of meaningful hand gestures, but not meaningless hand gestures, compared to stimulation over the vertex control site. We expected PV to increase in this scenario, considering that in a previous experiment (Reader et al. 2018a) we observed that when participants imitate meaningless actions, their wrist moves with greater velocity, possibly to increase the correction time available prior to the final hand posture formation. We reasonably expected that interrupting the activity of an area involved in action recognition could lead participants to take an approach to imitation more like that observed for meaningless actions. However, whilst our main effects of action meaning were in keeping with previous findings, we did not observe any influence of rTMS over pMTG on these correction time markers.

In the light of a failure to support our original hypothesis, what might the effect of rTMS over the left pMTG 
represent? One possibility is that stimulation over the left pMTG during action observation did in fact interfere with action recognition, which in turn introduced a delay in the following imitative action performance. This possibility is supported by meta-analytic evidence suggesting that the pMTG is more frequently associated with action observation than action performance (Caspers et al. 2010; Hardwick et al. 2018; Grèzes and Decety 2001), or proposals that this region processes observed action kinematics (Hamilton 2008). However, the fact that this effect was observed solely for meaningful hand gestures, and not for finger gestures (even when directly assessing the movement of the digits, rather than the wrist), is harder to explain.

Previous discussions regarding the different requirements of hand and finger gesture imitation have generally occurred in the context of meaningless action imitation deficits in apraxia. These skills have frequently been assessed using established hand and finger gesture stimuli (Goldenberg 1996). In particular, these hand gestures rely on positioning the hand relative to other parts of the body, which may require breaking down the observed gesture into basic spatial relationships between body parts (Goldenberg 2001; Goldenberg and Karnath 2006). In contrast, the finger gestures require serial positioning of the digits with the hand position remaining consistent. Some have suggested that these tasks are strongly reliant on different areas of the brain (Goldenberg and Karnath 2006; Goldenberg and Randerath 2015; Goldenberg 2001, 2009), whilst others propose a shared network (Achilles et al. 2017). Our hand and finger gestures were not designed in keeping with these classical distinctions, and the focus on meaningless action in previous reports makes it hard for us to draw parallels with existing neuropsychological work (but see Achilles et al. 2016). However, given that our hand gesture stimuli clearly show greater wrist position variability compared to the finger gesture stimuli, it is feasible the effect of rTMS over the left pMTG on wrist velocity has something to do with the postural aspects of the imitation task (i.e., the hand position relative to other parts of the body).

One interesting possibility is that a residual effect of the stimulation performed during action observation may have reduced the efficiency with which participants could use stored information regarding familiar (meaningful) hand postures during action. This would be in keeping with some previous neuroimaging work (e.g., Astafiev et al. 2004; Dinstein et al. 2007; Gallivan et al. 2016; Johnson-Frey et al. 2005; Lingnau and Downing 2015; Króliczak and Frey 2009; Oosterhof et al. 2010), and also suggestions from neuropsychological reports that the left posterior temporal lobe is involved in transitive movements in an imitative scenario or to demand (Buxbaum et al. 2014; Tarhan et al. 2015; but see; Vingerhoets and Clauwaert 2015). Notably, some have suggested that the left posterior temporal lobe is important for 'the production of postural aspects of tool-related actions' (Buxbaum et al. 2014, p. 1981). Our results suggest that this capacity may extend to intransitive, emblematic action performance. Whilst the left posterior temporal lobe may also be involved in the kinematic aspects of gesture (Buxbaum et al. 2014), it is important to note that our observed change in PV following rTMS over the pMTG may not be due to direct interference with kinematic processing. Rather, the reduced movement speed may reflect reduced certainty regarding the final hand position when the retrieval of postural information is impaired following stimulation.

The absence of TMS-related effects in finger gesture performance could be because our dataset or analysis may have been more sensitive to detect differences in hand gestures than in finger gestures. For example, if our effects of rTMS are due to interference with postural production, the postural components of finger gestures (i.e., their relative positions during gesture formation or at gesture completion) may not have been adequately captured by our stimuli or analysis. Alternatively, it could be that the organisation of semantic information regarding emblematic hand and finger gestures is segregated in such a way that our stimulation only interfered with hand gestures. The categorical organisation of information in occipitotemporal regions (Bracci et al. 2010, 2015, 2017; Downing et al. 2007; Lingnau and Downing 2015; Wurm and Lingnau 2015) might support this, though we are not convinced that our neuro-navigation approach is specific enough for such an effect.

\section{Imitation Accuracy}

As in a previous experiment (Reader and Holmes 2018), our actor-imitator correspondence analysis revealed that hand gestures were more accurately imitated (i.e., with greater correlation between actor and imitator velocity profiles) compared to finger gestures. The absence of TMS siterelated effects in this actor-imitator correspondence suggests that rTMS may not be sufficiently disruptive to influence imitation accuracy, compared to the deficits that can be observed following large scale lesion damage. This is also in keeping with what we have observed with similar experimental methods (Reader and Holmes 2018; Reader et al. 2018b), which suggest that rTMS over areas associated with imitation results in relatively subtle changes in kinematics, rather than changes in imitation accuracy as assessed by the correlation between actor and imitator velocity profiles.

\section{Effects of Action Meaning and Effector on Wrist Kinematics}

Finally, wrist kinematics showed similar effects of action meaning as previously reported. Specifically, meaningless 
actions had a significantly smaller TPV/MT and TPD/MT, along with a significantly greater PV and MT compared to meaningful actions (i.e., the correction time markers reported in Reader et al. 2018a).

Furthermore, we observed that meaningless hand gestures had a significantly greater PV than meaningful hand gestures, whilst meaningful and meaningless finger gestures were not significantly different. We also found that TPV/ MT was significantly smaller in meaningless compared to meaningful hand gestures, whilst MT was significantly longer in meaningless hand compared to meaningful hand gestures. As with PV, similar statistically significant effects were not observed for finger gestures. This seems to suggest that correction time strategies in meaningless actions are better captured in our hand gesture stimuli, perhaps because of the greater distance that the hand must move (mean $\pm \mathrm{SE}$ distance moved for wrist tracker was $26.9 \pm 1.10 \mathrm{~cm}$ for hand gestures, $18.9 \pm 0.725 \mathrm{~cm}$ for finger gestures). It might be useful in future to assess whether similar kinematic markers can be observed in the digits during finger gesture formation.

\section{Conclusion}

Our results provide causal evidence in healthy individuals for a role of the left pMTG in the imitation of meaningful (emblematic) hand gestures, which may support a role for the left posterior temporal lobe in producing known postural configurations (i.e., placing the hand relative to other parts of the body). However, more work is needed to better clarify this, and also better examine interactions between action meaning and action effector during imitation.

Acknowledgements We would like to thank Silvia Zeni for her invaluable assistance with data collection. This research was supported by the Economic and Social Research Council (Grant Number ES/J500148/1).

Open Access This article is distributed under the terms of the Creative Commons Attribution 4.0 International License (http://creativeco mmons.org/licenses/by/4.0/), which permits unrestricted use, distribution, and reproduction in any medium, provided you give appropriate credit to the original author(s) and the source, provide a link to the Creative Commons license, and indicate if changes were made.

\section{References}

Achilles EIS, Fink GR, Fischer MH, Dovern A, Held A, Timpert DC, Schroeter C, Schuetz K, Kloetzsch C, Weiss PH (2016) Effect of meaning on apraxic finger imitation deficits. Neuropsychologia 82:74-83. https://doi.org/10.1016/j.neuropsychologi a.2015.12.022

Achilles EIS, Weiss PH, Fink GR, Binder E, Price CJ, Hope TMH (2017) Using multi-level Bayesian lesion-symptom mapping to probe the body-part-specificity of gesture imitation skills.
NeuroImage 161:94-103. https://doi.org/10.1016/j.neuroimage .2017.08.036

Astafiev SV, Stanley CM, Shulman GL, Corbetta M (2004) Extrastriate body area in human occipital cortex responds to the performance of motor actions. Nat Neurosci 7(5):542-548

Binder JR, Desai RH, Graves WW, Conant LL (2009) Where is the semantic system? A critical review and meta-analysis of 120 functional neuroimaging studies. Cereb Cortex 19:2767-2796. https:// doi.org/10.1093/cercor/bhp055

Bracci S, Ietswaart M, Peelen MV, Cavina-Pratesi C (2010) Dissociable neural responses to hands and non-hand body parts in human left extrastriate visual cortex. J Neurophysiol 103(6):3389-3397. https ://doi.org/10.1152/jn.00215.2010

Bracci S, Caramazza A, Peelen MV (2015) Representational similarity of body parts in human occipitotemporal cortex. J Neurosci 35(38):12977-12985. https://doi.org/10.1523/JNEUR OSCI.4698-14.2015

Bracci S, Ritchie JB, Op de Beek, H (2017) On the partnership between neural representations of object categories and visual features in the ventral visual pathway. Neuropsychologia 105:153-164. https ://doi.org/10.1016/j.neuropsychologia.2017.06.010

Buxbaum LJ, Randerath J (2018) Limb apraxia and the left parietal lobe. Handb Clin Neurol 151:349-363. https://doi.org/10.1016/ B978-0-444-63622-5.00017-6

Buxbaum LJ, Shapiro AD, Coslett HB (2014) Critical brain regions for tool-related and imitative actions: a componential analysis. Brain 137:1971-1985. https://doi.org/10.1093/brain/awu111

Carmo JC, Rumiati RI (2009) Imitation of transitive and intransitive actions in healthy individuals. Brain Cognit 69:460-464. https:// doi.org/10.1016/j.bandc.2008.09.007

Caspers S, Zilles K, Laird AR, Eickhoff SB (2010) ALE meta-analysis of action observation and imitation in the human brain. NeuroImage 50:1148-1167. https://doi.org/10.1016/j.neuroimage .2009 .12 .112

Davey J, Cornelissen PL, Thompson HE, Sonkusare S, Hallam G, Smallwood J, Jefferies E (2015) Automatic and controlled semantic retrieval: TMS reveals distinct contributions of posterior middle temporal gyrus and angular gyrus. J Neurosci 35(46):15230 15239. https://doi.org/10.1523/JNEUROSCI.4705-14.2015

Decety J, Grèzes J, Costes N, Perani D, Jeannerod M, Procyk E, Grassi F, Fazio F (1997) Brain activity during observation of actions: influence of action content and subject's strategy. Brain 120:1763-1777

Dinstein I, Hasson U, Rubin N, Heeger DJ (2007) Brain areas selective for both observed and executed movements. J Neurophysiol 98:1415-1427. https://doi.org/10.1152/jn.00238.2007

Downing PE, Wiggett AJ, Peelen MV (2007) Functional magnetic resonance imaging investigation of overlapping lateral occipitotemporal activations using multi-voxel pattern analysis. J Neurosci 27:226-233. https://doi.org/10.1523/JNEUROSCI.3619-06.2007

Gallivan JP, Johnsrude IS, Flanagan JR (2016) Planning ahead: objectdirected sequential actions decoded from human frontoparietal and occipitotemporal networks. Cereb Cortex 26(2):708-730. https://doi.org/10.1093/cercor/bhu302

Goldenberg G (1996) Defective imitation of gestures in patients with damage in the left or right hemispheres. J Neurol Neurosurg Psychiatry $61: 176-180$

Goldenberg G (2001) Imitation and matching of hand and finger postures. NeuroImage 14:S132-S136. https://doi.org/10.1006/ nimg. 2001.0820

Goldenberg G (2009) Apraxia and the parietal lobes. Neuropsychologia 47:1449-1459. https://doi.org/10.1016/j.neuropsychologi a.2008.07.014

Goldenberg G, Karnath H (2006) The neural basis of imitation is body part specific. J Neurosci 26:6282-6287. https://doi.org/10.1523/ JNEUROSCI.0638-06.2006 
Goldenberg G, Randerath J (2015) Shared neural substrates of apraxia and aphasia. Neuropsychologia 75:40-49. https://doi. org/10.1016/j.neuropsychologia.2015.05.017

Grèzes J, Decety J (2001) Functional anatomy of execution, mental simulation, observation, and verb generation of actions: a metaanalysis. Hum Brain Mapp 12:1-19

Hamilton AF de C (2008) Emulation and mimicry for social interaction: a theoretical approach to imitation in autism. Q J Exp Psychol 6(1):101-115. https://doi.org/10.1080/17470210701508798

Hardwick RM, Caspers S, Eickhoff SB, Swinnen SP (2018) Neural correlates of action: comparing meta-analyses of imagery, observation, and execution. Neurosci Biobehav Rev 94:31-44. https:// doi.org/10.1016/j.neubiorev.2018.08.003

Johnson-Frey SH, Newman-Norlund R, Grafton ST (2005) A distributed left hemisphere network active during planning of everyday tool use skills. Cereb Cortex 15(6):681-695

Kalénine S, Buxbaum LJ, Coslett HB (2010) Critical brain regions for action recognition: lesion symptom mapping in left hemisphere stroke. Brain 133:3269-3280. https://doi.org/10.1093/brain/awq21 0

Króliczak G, Frey SH (2009) A common network in the left cerebral hemisphere represents planning of tool use pantomimes and familiar intransitive gestures at the hand-dependent level. Cereb Cortex 19:2396-2410. https://doi.org/10.1093/cercor/bhn261

Kubiak A, Króliczak G (2016) Left extrastriate body area is sensitive to the meaning of symbolic gesture: evidence from fMRI repetition suppression. Sci Rep 6:31064. https://doi.org/10.1038/srep31064

Lingnau A, Downing PE (2015) The lateral occipitotemporal cortex in action. Trends Cogn Sci 19(5):268-277. https://doi.org/10.1016/j. tics.2015.03.006

Menz MM, McNamara A, Klemen J, Binkofski F (2009) Dissociating networks of imitation. Hum Brain Mapp 30:3339-3350. https:// doi.org/10.1002/hbm.20756

Möttönen R, Farmer H, Watkins KE (2016) Neural basis of understanding communicative actions: changes associated with knowing the actor's intention and the meaning of actions. Neuropsychologia 81:230-237. https://doi.org/10.1016/j.neuropsychologi a.2016.01.002

Oosterhof NN, Wiggett AJ, Diedrichsen J, Tipper SP, Downing PE (2010) Surface-based information mapping reveals crossmodal vision-action representations in human parietal and occipitotemporal cortex. J Neurophysiol 104:1077-1089. https://doi. org/10.1152/jn.00326.2010

Peigneux P, Van der Linden M, Garraux G, Laureys S, Degueldre C, Aerts J, Del Fiore G, Moonen G, Luxen A, Salmon E (2004) Imaging a cognitive model of apraxia: the neural substrate of gesture-specific cognitive processes. Hum Brain Mapp 21:119-142. https://doi.org/10.1002/hbm.10161

Petreska B, Adriani M, Blanke O, Billard AG (2007) Apraxia: a review. Prog Brain Res 164:61-83. https://doi.org/10.1016/S0079 -6123(07)64004-7

Press C, Heyes C (2008) Stimulus-driven selection of routes to imitation. Exp Brain Res 188:147-152. https://doi.org/10.1007/s0022 1-008-1422-9

Reader AT, Holmes NP (2018) The left ventral premotor cortex is involved in hand shaping for intransitive gestures: evidence from a two-person imitation experiment. R Soc Open Sci 5(10): 181356. https://doi.org/10.1098/rsos.181356

Reader AT, Rao VM, Christakou A, Holmes NP (2018a) A kinematic examination of dual-route processing for action imitation. Atten Percept Psychophys. https://doi.org/10.3758/s13414-018-1582-z
Reader AT, Royce BP, Marsh JE, Chivers K, Holmes NP (2018b) Repetitive transcranial magnetic stimulation reveals a role for the left inferior parietal lobule in matching observed kinematics during imitation. Eur J Neurosci 47(8):918-928. https://doi. org/10.1111/ejn.13886

Rossi S, Hallett M, Rossini PM, Pascual-Leone A, The Safety of TMS Consensus Group (2009) Safety, ethical considerations, and application guidelines for the use of transcranial magnetic stimulation in clinical practice and research. Clin Neurophysiol 120:2008 2039. https://doi.org/10.1016/j.clinph.2009.08.016

Rossini PM, Barker AT, Berardelli A, Caramia MD, Caruso G, Cracco RQ, Dimitrijević MR, Hallett M, Katayama Y, Lücking CH et al (1994) Non-invasive electrical and magnetic stimulation of the brain, spinal cord and roots: basic principles and procedures for routine clinical application. Report of an IFCN committee. Electroencephalogr Clin Neurophysiol 91:79-92

Rumiati RI, Weiss PH, Tessari A, Assmus A, Zilles K, Herzog H, Fink GR (2005) Common and differential neural mechanisms supporting imitation of meaningful and meaningless actions. J Cogn Neurosci 17:1420-1431. https://doi.org/10.1162/0898929054985374

Rumiati RI, Carmo JC, Corradi-Dell'Acqua C (2009) Neuropsychological perspectives on the mechanisms of imitation. Philos Trans R Soc B 364:2337-2347. https://doi.org/10.1098/rstb.2009.0063

Stokes MG, Chambers CD, Gould IC, English T, McNaught E, McDonald O, Mattingley JB (2007) Distance-adjusted motor threshold for transcranial magnetic stimulation. Clin Neurophysiol 118:1617-1625. https://doi.org/10.1016/j.clinph.2007.04.004

Tanaka S, Inui T, Iwaki S, Konishi J, Nakai T (2001) Neural substrates involved in imitating finger configurations: an fMRI study. NeuroReport 12:1171-1174

Tarhan LY, Watson CE, Buxbaum LJ (2015) Shared and distinct neuroanatomic regions critical for tool-related action production and recognition: evidence from 131 left-hemisphere stroke patients. J Cogn Neurosci 27(12):2491-2511. https://doi.org/10.1162/ jocn_a_00876

Tessari A, Cubelli R (2014) Route selection in action imitation: a matter of strategic choice? Cortex 57:277-278. https://doi. org/10.1016/j.cortex.2014.01.014

Tessari A, Rumiati RI (2004) The strategic control of multiple routes in imitation of actions. J Exp Psychol Hum Percept Perform 30:1107-1116. https://doi.org/10.1037/0096-1523.30.6.1107

Villarreal M, Fridman EA, Amengual A, Falasco G, Gerscovich ER, Ulloa ER, Leiguarda RC (2008) The neural substrate of gesture recognition. Neuropsychologia 46:2371-2382. https://doi. org/10.1016/j.neuropsychologia.2008.03.004

Vingerhoets G, Clauwaert A (2015) Functional connectivity associated with hand shape generation: imitating novel hand postures and pantomiming tool grips challenge different nodes of a shared neural network. Hum Brain Mapp 36:3426-3440. https://doi. org/10.1002/hbm.22853

Wurm MF, Lingnau A (2015) Decoding actions at different levels of abstraction. J Neurosci 35(20):7727-7735. https://doi. org/10.1523/JNEUROSCI.0188-15

Wurm MF, Caramazza A, Lingnau A (2017) Action categories in lateral occipitotemporal cortex are organized along sociality and transitivity. J Neurosci 37(3):562-575. https://doi.org/10.1523/ JNEUROSCI.1717-16.2016 